\title{
Relationship between aeration and rheology of breads.
}

\begin{abstract}
The relationship between aeration and rheology of bread was investigated by varying ingredient and processing factors during bread proofing and baking processes. Dough and bread aeration were quantified using density measurements from the volumetric methods. Dough rheology was characterized by the rising rate and maximum proofed height under a constant force using a texture analyzer, while bread rheology was characterized using the bread firmness test. Increasing flour strength and yeast level decreased dough and bread density and bread firmness but increased the rising rate and maximum proofed height of dough significantly $(\mathrm{P}<0.02)$. Proofing time had no effect on dough's rising rate, although it decreased dough and bread density $(\mathrm{P}<0.01)$ and bread firmness $(\mathrm{P}<0.002)$ and increased dough maximum proofed height $(\mathrm{P}<0.02)$. Baking temperature did not show significant effect on bread density $(\mathrm{P}<0.06)$ and had no effect on bread firmness. Dough and bread densities gave good correlations $(\mathrm{R} 2>0.79)$ with its rheological properties.
\end{abstract}

Keyword: Aeration; Bread; Density; Dough; Firmness; Rheology. 